\title{
Cyclic triangle factors in regular tournaments
}

\author{
Lina Li \\ Department of Mathematics \\ University of Illinois at Urbana-Champaign \\ Urbana, Illinois, U.S.A. \\ linali2@illinois.edu
}

\author{
Theodore Molla* \\ Department of Mathematics and Statistics \\ University of South Florida \\ Tampa, Florida, U.S.A \\ molla@usf.edu
}

Submitted: Mar 30, 2018; Accepted: Sep 4, 2019; Published: Oct 25, 2019

(c) The authors. Released under the CC BY-ND license (International 4.0).

\begin{abstract}
Both Cuckler and Yuster independently conjectured that when $n$ is an odd positive multiple of 3 every regular tournament on $n$ vertices contains a collection of $n / 3$ vertex-disjoint copies of the cyclic triangle. Soon after, Keevash \& Sudakov proved that if $G$ is an orientation of a graph on $n$ vertices in which every vertex has both indegree and outdegree at least $(1 / 2-o(1)) n$, then there exists a collection of vertex-disjoint cyclic triangles that covers all but at most 3 vertices. In this paper, we resolve the conjecture of Cuckler and Yuster for sufficiently large $n$.
\end{abstract}

Mathematics Subject Classifications: 05C70, 05C20, 05C38

\section{Introduction}

Let $H$ and $G$ be graphs or directed graphs. An $H$-tiling of $G$ is a collection of vertexdisjoint copies of $H$ in $G$. An $H$-tiling $\mathcal{C}$ covers the set $V(\mathcal{C})=\bigcup_{C \in \mathcal{C}} V(C)$, and is called perfect or an $H$-factor if it covers $V(G)$.

The celebrated Hajnal-Szemerédi Theorem [7] states that for every positive integer $r$, if $n$ is a positive multiple of $r$ and $G$ is a graph on $n$ vertices such that $\delta(G) \geqslant(1-1 / r) n$, then $G$ contains a $K_{r}$-factor. The case when $r=3$ is a corollary of an earlier result of Corrádi \& Hajnal [3].

In this paper, we consider a similar problem in the context of oriented graphs, which are orientations of simple graphs, i.e., oriented graphs are directed graphs in which there is at most one directed edge between every pair of vertices and no loops. A tournament is an orientation of a complete graph. For an oriented graph $G$ and $v \in V(G)$, we denote the out-neighborhood of $v$ and in-neighborhood of $v$ by $N^{+}(v)$ and $N^{-}(v)$, respectively. We let $N(v)=N^{+}(v) \cup N^{-}(v)$ be the neighborhood of $v$, and we let $d^{+}(v)=\left|N^{+}(v)\right|$

\footnotetext{
*Research was partially supported by NSF Grant DMS-1500121 and DMS-1800761.
} 
and $d^{-}(v)=\left|N^{-}(v)\right|$ be the outdegree and indegree of $v$, respectively. The minimum semidegree of $G$ is

$$
\delta^{0}(G)=\min _{v \in V(G)}\left\{\min \left\{d^{+}(v), d^{-}(v)\right\}\right\} .
$$

An oriented graph $G$ on $n$ vertices is a regular tournament if $d^{+}(v)=d^{-}(v)=\frac{n-1}{2}$ for every $v \in G$.

A tournament is transitive if it contains no directed cycles, and the unique transitive tournament on $r$ vertices is denoted $T T_{r}$. Up to isomorphism, there are two different tournaments on three vertices: $T T_{3}$ and the three vertex cycle in which the edges are consistently oriented, which we denote by $C_{3}$. We call $C_{3}$ and $T T_{3}$ the cyclic and transitive triangles, respectively.

There has been some prior work on minimum degree conditions that force an $H$-factor in directed graphs. See [22] and [6] for work on directed graphs, and [1] and [23] for oriented graphs. Also, [21] contains many additional interesting embedding problems for oriented graphs. This paper focuses on the following conjecture that Cuckler and Yuster made independently.

Conjecture 1 (Cuckler 2008 [5], Yuster 2007 [24]). If $n$ is an odd positive multiple of 3, then every regular tournament on $n$ vertices has a cyclic triangle factor.

Keevash \& Sudakov then proved the following approximate version of this conjecture.

Theorem 2 (Keevash \& Sudakov 2009 [13]). There exists $c>0$ and $n_{0}$ such that for every $n \geqslant n_{0}$ the following holds. If $G$ is an oriented graph on $n$ vertices and $\delta^{0}(G) \geqslant(1 / 2-c) n$, then there exists a cyclic triangle tiling that covers all but at most 3 vertices.

A corollary of our main result resolves Conjecture 1 for large tournaments. To see that the resolution of Conjecture 1 is a sharp result, consider the following construction from [13]. For a positive integer $m$, let $G$ be a tournament on $3 m$ vertices in which the edges are oriented so that there exists a partition $\left\{V_{1}, V_{2}, V_{3}\right\}$ of $V(G)$ such that

- $\left|V_{1}\right|=m-1,\left|V_{2}\right|=m$, and $\left|V_{3}\right|=m+1 ;$

- for $i \in[3]$, the oriented graph induced by $V_{i}$ has minimum semidegree $\left\lfloor\left(\left|V_{i}\right|-1\right) / 2\right\rfloor$; and

- no edges are directed from $V_{2}$ to $V_{1}$, from $V_{3}$ to $V_{2}$, and from $V_{1}$ to $V_{3}$.

We have that,

$$
\delta^{0}(G)= \begin{cases}\left(\left|V_{2}\right|-2\right) / 2+\left|V_{1}\right|=\frac{n-4}{2} & \text { if } m \text { is even } \\ \left(\left|V_{2}\right|-1\right) / 2+\left|V_{1}\right|=\frac{n-3}{2} & \text { if } m \text { is odd } .\end{cases}
$$

To see why $G$ has no cyclic triangle factor, let $\mathcal{C}$ be a cyclic triangle tiling of $G$ and note that, for every $C \in \mathcal{C}$, either $C$ has one vertex in each of $V_{1}, V_{2}$ and $V_{3}$, or $V(C) \subseteq V_{i}$ for some $i \in[3]$. Therefore,

$$
\left|V(\mathcal{C}) \cap V_{1}\right| \equiv\left|V(\mathcal{C}) \cap V_{2}\right| \equiv\left|V(\mathcal{C}) \cap V_{3}\right| \quad(\bmod 3) .
$$


Because $\left|V_{1}\right|,\left|V_{2}\right|$ and $\left|V_{3}\right|$ are distinct modulo 3 , we have that $V(\mathcal{C}) \neq V(G)$. Motivated by this example we make the following definitions.

Definition 3 (Divisibility barrier and $\gamma$-extremal). Let $G$ be an oriented graph. Call a partition $\mathcal{P}$ of $V(G)$ a divisibility barrier if either $\mathcal{P}$ is the trivial partition $\{V(G)\}$ and $|V(G)|$ is not divisible by 3 , or if $\mathcal{P}$ has exactly three parts, $V_{1}, V_{2}$, and $V_{3}$, such that there are no edges directed from $V_{2}$ to $V_{1}$, from $V_{3}$ to $V_{2}$, and from $V_{1}$ to $V_{3}$; and $\left|V_{1}\right|,\left|V_{2}\right|$, and $\left|V_{3}\right|$ are not all equivalent modulo 3 .

For $\gamma>0$, call a partition $\left\{V_{1}, V_{2}, V_{3}\right\}$ of $V(G)$ a $\gamma$-extremal partition of $G$ if, for every $i \in[3]$,

$$
(1 / 3-\gamma) n \leqslant\left|V_{1}\right|,\left|V_{2}\right|,\left|V_{3}\right| \leqslant(1 / 3+\gamma) n,
$$

and the number of edges directed from $V_{2}$ to $V_{1}$, from $V_{3}$ to $V_{2}$, and from $V_{1}$ to $V_{3}$ are each at most $\gamma n^{2}$. An oriented graph is called $\gamma$-extremal if it contains a $\gamma$-extremal partition.

The following is the main result of this paper.

Theorem 4. There exists $c>0$ and $n_{0}$ such that for every $n \geqslant n_{0}$ and for every oriented graph $G$ on $n$ vertices with $\delta^{0}(G) \geqslant(1 / 2-c) n$ the following holds. G has a cyclic triangle factor if and only if $G$ does not have a divisibility barrier.

The following corollary resolves Cuckler and Yuster's conjecture for sufficiently large regular tournaments.

Corollary 5. There exists $n_{0}$ such that when $n$ is a multiple of 3 and $n \geqslant n_{0}$ the following holds. If $G$ is an oriented graph on $n$ vertices and $\delta^{0}(G) \geqslant n / 2-1$, then $G$ has a cyclic triangle factor.

Proof. Assuming that Theorem 4 holds, we only need to show that $G$ does not contain a divisibility barrier. For a contradiction, assume that $\left\{V_{1}, V_{2}, V_{3}\right\}$ is a divisibility barrier. Since $n$ is divisible by 3 and $\left|V_{1}\right|,\left|V_{2}\right|$, and $\left|V_{3}\right|$ are not all equivalent modulo 3 , we have that $\left|V_{1}\right|,\left|V_{2}\right|$ and $\left|V_{3}\right|$ are distinct modulo 3. Therefore, there exists a labeling $\{i, j, k\}=[3]$ such that $\left|V_{j}\right| \leqslant\left|V_{k}\right|-2$. Suppose that $i+1 \equiv j(\bmod 3)$. There exists $v \in V_{i}$ such that $\left|N^{+}(v) \cap V_{i}\right|<\left|V_{i}\right| / 2$, so

$$
d^{+}(v)=\sum_{\ell=1}^{3}\left|N^{+}(v) \cap V_{\ell}\right|<\frac{\left|V_{i}\right|}{2}+\left|V_{j}\right| \leqslant \frac{\left|V_{i}\right|+\left|V_{j}\right|+\left|V_{k}\right|-2}{2}=\frac{n}{2}-1,
$$

a contradiction. A similar argument holds when $i-1 \equiv j(\bmod 3)$.

We are not sure how large the constant $c$ can be in Theorem 4, and we do not compute the value of $c$ that our proof implies. An example of Keevash \& Sudakov [13], which we will present below, implies that the constant $c$ cannot be larger than $1 / 18$. This suggests the following problem.

Problem 6. What is the smallest $\phi \geqslant 0$ such that there exists $n_{0}$ such that for every $n \geqslant n_{0}$ every oriented graph $G$ on $n$ vertices with $\delta^{0}(G) \geqslant(4 / 9+\phi) n$ contains either a divisibility barrier or a cyclic triangle factor? 
For $m \geqslant 1$, let $G$ be an oriented graph on $n=9(m+1)$ vertices and let $\mathcal{P}=\left\{V_{1}, V_{2}, V_{3}\right\}$ be a partition of $V(G)$ such that $\left|V_{1}\right|=3 m+1$ and $\left|V_{2}\right|=\left|V_{3}\right|=3 m+4$. Suppose that for every pair $i, j \in[3]$ such that $j \equiv i+1(\bmod 3)$, there is a directed edge from every vertex in $V_{i}$ to every vertex in $V_{j}$. Further suppose that, for every $i \in[3]$, the vertices of $V_{i}$ can be cyclically ordered so that, for every $v \in V_{i}$, the intersection of the out-neighborhood of $v$ and $V_{i}$ is exactly the $\left(\left|V_{i}\right|-1\right) / 3$ vertices that succeed $v$ in this ordering. Note that every cyclic triangle has at least one vertex in $V_{1}$, so $|\mathcal{C}| \leqslant\left|V_{1}\right|=n / 3-2$ for every cyclic triangle tiling $\mathcal{C}$, and

$$
\delta^{0}(G)=\frac{\left|V_{2}\right|-1}{3}+\left|V_{1}\right|=\frac{4 n}{9}-2 .
$$

Note that $\mathcal{P}$ is not a divisibility barrier, because the three parts of $\mathcal{P}$ all have the same size modulo 3. Additionally, $G$ cannot have a divisibility barrier, because for every partition $\mathcal{P}^{\prime} \neq \mathcal{P}$, such that $\left|\mathcal{P}^{\prime}\right| \leqslant 3$, there exists a part $U \in \mathcal{P}^{\prime}$ such that there exist $x, y \in U$ such that $x$ and $y$ are in different parts, say $V_{i}$ and $V_{j}$, of $\mathcal{P}$. But then either $\mathcal{P}^{\prime}$ is not a divisibility barrier or $U$ contains all of the vertices of the third part, say $V_{k}$, of $\mathcal{P}$. Then, by similar logic, one can argue that either $\mathcal{P}^{\prime}$ is not a divisibility barrier or $U=V(G)$. Since 3 divides $|V(G)|, \mathcal{P}^{\prime}$ is not a divisibility barrier.

If, for some $\psi>1 / 3$ and all sufficiently large $n$, there exists a $C_{3}$-free oriented graph on $n$ vertices with minimum semidegree $\psi n$, then a similar example would imply that $\phi$ must be strictly greater than 0 in Problem 6 . Note that such a $C_{3}$-free oriented graph would imply that the famous Caccetta-Häggkvist Conjecture [2] is false. ${ }^{1}$

\subsection{Additional Definitions and Notation}

Let $G$ be an oriented graph, $u, v \in V(G)$, and $A, B \subseteq V(G)$. We let $N^{+}(u)$ be the out-neighborhood of $u, N^{+}(u, B)=N^{+}(u) \cap B, d^{+}(u, B)=\left|N^{+}(u, B)\right|$, and $e^{+}(A, B)=$ $\sum_{u \in A} d^{+}(u, B)$. We define $N^{-}(u), N^{-}(u, B), d^{-}(u, B)$ and $e^{-}(A, B)$ similarly. If $u v \in$ $E(G)$, we let $d^{+,-}(u v, A)=\left|N^{+}(u) \cap N^{-}(v) \cap A\right|$ and $d^{-,+}(u v, A), d^{+,+}(u v, A)$, and $d^{-,-}(u v, A)$ are all defined similarly. We also let $d^{\sigma, \tau}(u v)=d^{\sigma, \tau}(u v, V(G))$, for $\sigma, \tau \in$ $\{-,+\}$. We let $E(A)$ be the set of edges in the oriented graph induced by $A$, and let $e(A)=|E(A)|$. We let $\bar{A}=V(G) \backslash A$.

We define $E^{+}(A, B)=\{u v \in E(G): u \in A$ and $v \in B\}$ and $E^{-}(A, B)=E^{+}(B, A)$. We will often write cyclic and transitive triangles $C$ as $a b c$ when $V(C)=\{a, b, c\}$. For $V_{1}, V_{2}, V_{3} \subseteq V(G), \operatorname{cyc}\left(V_{1}, V_{2}, V_{3}\right)$ and $\operatorname{trn}\left(V_{1}, V_{2}, V_{3}\right)$ count, respectively, the number of cyclic and transitive triangles with vertex set $\left\{v_{a}, v_{b}, v_{c}\right\}$ such that $\{a, b, c\}=[3]$ and $v_{i} \in V_{i}$ for $i \in[3]$. We abbreviate $\operatorname{cyc}(A, A, A)$ and $\operatorname{trn}(A, A, A)$ as $\operatorname{cyc}(A)$ and $\operatorname{trn}(A)$, respectively. We will often replace $\{v\}$ with $v$ in this notation.

We define the strong $\beta$-out-neighborhood of $A$ to be the set of vertices $x \in \bar{A}$ such that $d^{-}(x, A) \geqslant|A|-\beta n$ and we denote this set by $\mathrm{SN}_{\beta}^{+}(A)$. We define the strong $\beta$-in-neighborhood $\mathrm{SN}_{\beta}^{-}(A)$ of $A$ similarly.

\footnotetext{
${ }^{1}$ If true, the Cacceta-Häggkvist Conjecture would imply that every oriented graph on $n$ vertices with minimum outdegree at least $n / 3$ contains a cyclic triangle.
} 
Throughout the paper, we write $0<\alpha \ll \beta \ll \gamma$ to mean that we can choose the constants $\alpha, \beta, \gamma$ from right to left. More precisely, there are increasing functions $f$ and $g$ such that, given $\gamma$, whenever we choose $\beta \leqslant f(\gamma)$ and $\alpha \leqslant g(\beta)$, all calculations needed in our proof are valid. Hierarchies of other lengths are defined in the obvious way. For real numbers $x$ and $y$, we write $x=y \pm c$ to mean that $y-c \leqslant x \leqslant y+c$.

In our proof, we use a very small part of the theory and notation developed in [11], [10],[8],[15], and [17]. It is based on the absorbing method of Rödl, Ruciński and Szemerédi [20]. This theory was developed for hypergraphs, so we define, for every oriented graph $G$, the hypergraph $H(G)$ to be the 3-uniform hypergraph in which $x y z$ is in an edge if and only if $x y z$ is a cyclic triangle in $G$. Clearly a cyclic triangle factor in $G$ is equivalent to a perfect matching in $H(G)$.

Let $V$ be a set of order $n$ and let $\mathcal{P}=\left\{V_{1}, \ldots, V_{d}\right\}$ be a partition of $V$. We say that $\mathcal{P}$ is trivial if $|\mathcal{P}|=1$, and, for $\eta>0$, we call $\mathcal{P}$ an $\eta$-partition if $\left|V_{i}\right| \geqslant \eta n$ for every $i \in[d]$. Let $H$ be a $k$-uniform hypergraph with vertex set $V$. We let

$$
\delta_{1}(H)=\min _{v \in V}\{|\{e \in E(H): v \in e\}|\} .
$$

For every subset $U$ of $V$ the index vector with respect to $\mathcal{P}$, denoted $\mathbf{i}_{\mathcal{P}}(U)$, is the vector defined by

$$
\mathbf{i}_{\mathcal{P}}(U)=\left(\left|U \cap V_{1}\right|,\left|U \cap V_{2}\right|, \ldots,\left|U \cap V_{d}\right|\right) .
$$

Let $I_{\mathcal{P}}(H)=\left\{\mathbf{i}_{\mathcal{P}}(e): e \in E(H)\right\}$ be the set of edge-vectors and, for $\mu>0$, let

$$
I_{\mathcal{P}}^{\mu}(H)=\left\{\mathbf{v} \in I_{\mathcal{P}}(H): \text { there are at least } \mu n^{k} \text { edges } e \text { in } H \text { such that } \mathbf{v}=\mathbf{i}_{\mathcal{P}}(e)\right\},
$$

be the set of $\mu$-robust edge-vectors.

We call an additive subgroup of $\mathbb{Z}^{d}$ a lattice, and we let $L_{\mathcal{P}}(H)$ and $L_{\mathcal{P}}^{\mu}(H)$ be the lattices generated by $I_{\mathcal{P}}(H)$ and $I_{\mathcal{P}}^{\mu}(H)$, respectively. Clearly if $M$ is a collection of vertexdisjoint edges in $H$, then $\mathbf{i}_{\mathcal{P}}(V(M)) \in L_{\mathcal{P}}(H)$. Therefore, $H$ does not have a perfect matching if $\mathbf{i}_{\mathcal{P}}(V(H)) \notin L_{\mathcal{P}}(H)$. For example, suppose that $G$ is an oriented graph with a divisibility barrier. If $|V(G)|$ is not divisible by 3 , then $\mathbf{i}_{\mathcal{P}}(V(G)) \notin L_{\mathcal{P}}(H(G))$ when $\mathcal{P}=\{V(G)\}$. Otherwise, there exists a partition $\mathcal{P}=\left\{V_{1}, V_{2}, V_{3}\right\}$ of $V(G)$ such that the entries of $\mathbf{i}_{\mathcal{P}}(V(G))$ are each different modulo 3, and, for every $e \in E(H(G))$, the entries of $\mathbf{i}_{\mathcal{P}}(e)$ are each the same modulo 3 , so $\mathbf{i}_{\mathcal{P}}(V(G)) \notin L_{\mathcal{P}}(H(G))$. We let $\mathbf{u}_{i} \in \mathbb{Z}^{d}$ be the $i$ th unit vector, i.e., $\mathbf{u}_{i}$ is the vector in which the $i$ th component is 1 and every other component is 0 . A transferral is a vector $\mathbf{v}$ in $\mathbb{Z}^{d}$ such that $\mathbf{v}=\mathbf{u}_{i}-\mathbf{u}_{j}$ for distinct $i$ and $j$ in $[d]$. A 2-transferral $\mathbf{v} \in L_{\mathcal{P}}^{\mu}(H)$ is a transferral such that $\mathbf{v}=\mathbf{v}_{1}-\mathbf{v}_{2}$ for $\mathbf{v}_{1}, \mathbf{v}_{2} \in I_{\mathcal{P}}^{\mu}(H)$. We say that $L_{\mathcal{P}}^{\mu}(H)$ is 2-transferral-free if $L_{\mathcal{P}}^{\mu}(H)$ does not contain a 2-transferral.

Let $x$ and $y$ be vertices in $V$ and let $\beta>0$. A set $S \subseteq V$ is called an $(H, x, y)$-linking $(k \ell-1)$-set if both $H[S \cup\{x\}]$ and $H[S \cup\{y\}]$ have perfect matchings and $|S|=k \ell-1$. The vertices $x$ and $y$ are $(H, \beta, \ell)$-reachable if there are at least $\beta n^{\ell k-1}(H, x, y)$-linking $(k \ell-1)$-sets. A set $U \subseteq V$ is $(H, \beta, \ell)$-closed if every pair of distinct vertices $x$ and $y$ in $U$ is $(H, \beta, \ell)$-reachable. Call a partition $\mathcal{P}=\left\{V_{1}, \ldots, V_{d}\right\}$ of $V(H)$ a $(H, \beta, \ell)$-closed partition if, for every $i \in[d], V_{i}$ is $(H, \beta, \ell)$-closed. For every vertex $x \in V(H)$, let $\tilde{N}_{H}(\beta, \ell, x)$ be the set of vertices $y$ such that $x$ and $y$ are $(H, \beta, \ell)$-reachable. 


\section{Proof of Theorem 4}

\subsection{Overview}

Our proof follows the stability method, i.e., we divide the proof into two cases depending on whether $G$ is $\gamma$-extremal. The case when $G$ is $\gamma$-extremal is handled in the following lemma, the proof of which we defer until Section 2.5.

Lemma 7 (Extremal case). Suppose that $1 / n \ll c, \gamma \ll 1, n$ is divisible by 3 , and $G$ is an oriented graph on $n$ vertices. If $\delta^{0}(G) \geqslant(1 / 2-c) n$ and $G$ is $\gamma$-extremal, then $G$ has a cyclic triangle factor if and only if $G$ does not have a divisibility barrier.

The proof in the case when $G$ is not $\gamma$-extremal follows the absorbing method, and the following lemma of Lo \& Markström [17] serves as our absorbing lemma.

Lemma 8 (Absorbing Lemma - Lemma 1.1 in [17]). Given

$$
1 / n \ll \alpha \ll \eta \ll \beta \ll 1 / \ell, 1 / k
$$

the following holds. If $H$ is a k-uniform hypergraph on $n$ vertices and $V(H)$ is $(H, \beta, \ell)$ closed, then there exists a subset $U$ of $V(H)$ where $|U|$ is at most $\eta n$ and divisible by $k$ such that for every $W \subseteq V(H) \backslash U$ with $|W|$ at most $\alpha$ n and divisible by $k$ there exists a perfect matching of $H[U \cup W]$.

Lemma 8 and Theorem 2 imply the following lemma.

Lemma 9. Suppose that $1 / n \ll c \ll \beta \ll 1 / \ell, n$ is divisible by 3 , and that $G$ is an oriented graph on $n$ vertices. If $\delta^{0}(G) \geqslant(1 / 2-c) n$ and $V(G)$ is $(H(G), \beta, \ell)$-closed, then $G$ has a cyclic triangle factor.

Proof. Introduce constants $\eta$ and $\alpha$ so that

$$
1 / n \ll \alpha \ll \eta \ll c \ll \beta \ll 1 / \ell
$$

Let $U \subseteq V(G)$ be the set guaranteed by Lemma 8 and let $G^{\prime}=G-U$. Note that

$$
\delta^{0}\left(G^{\prime}\right) \geqslant \delta^{0}(G)-|U| \geqslant(1 / 2-c-\eta) n \geqslant(1 / 2-2 c)\left|G^{\prime}\right| .
$$

Therefore, by Theorem 2, with $2 c$ and $G^{\prime}$ playing the roles of $c$ and $G$, respectively, there exists a cyclic triangle tiling $\mathcal{C}$ of $G^{\prime}$ such that if $W=V\left(G^{\prime}\right) \backslash V(\mathcal{C})$, then $|W| \in\{0,3\}$. Since $|W| \leqslant \alpha n$ and $|W|$ is divisible by 3, there exists a triangle factor $\mathcal{C}^{\prime}$ of $G[U \cup W]$. Hence, $\mathcal{C} \cup \mathcal{C}^{\prime}$ is a triangle factor of $G$.

Note that, with Lemmas 7 and 9, the following lemma implies Theorem 4.

Lemma 10. Suppose that $1 / n \ll c \ll \beta \ll \gamma<1$ and that $G$ is an oriented graph on $n$ vertices. If $\delta^{0}(G) \geqslant(1 / 2-c) n$, and, for every positive integer $\ell \leqslant 1000$, we have that $V(G)$ is not $(H(G), \beta, \ell)$-closed, then $G$ is $\gamma$-extremal. 
The proof of Lemma 10 relies on the following four lemmas.

Lemma 11. Suppose that $1 / n \ll \beta \ll \beta^{\prime}, \mu \ll 1 / d, 1 / \ell, G$ is an oriented graph on $n$ vertices, and that $\mathcal{P}=\left\{V_{1}, \ldots, V_{d}\right\}$ is a partition of $V(G)$. If $\mathcal{P}$ is $\left(H(G), \beta^{\prime}, \ell\right)$-closed and there exists a 2-transferral $\mathbf{u}_{i}-\mathbf{u}_{j} \in L_{\mathcal{P}}^{\mu}(H(G))$, then the partition formed by merging $V_{i}$ and $V_{j}$ is $(H(G), \beta, 4 \ell+1)$-closed.

Lemma 12. Suppose that $1 / n \ll c \ll \beta \ll 1$ and that $G$ is an oriented graph on $n$ vertices such that $\delta^{0}(G) \geqslant(1 / 2-c) n$. Then there exists $\mathcal{P}=\left\{V_{1}, \ldots, V_{d}\right\}$ a $(H(G), \beta, 8)$-closed partition of $V(G)$ such that $d \leqslant 4$ and $\left|V_{i}\right|>n / 9$ for every $i \in[d]$.

Lemma 13. Suppose that $1 / n \ll c \ll \mu \ll \alpha \ll \eta<1$ and $G$ is an oriented graph on $n$ vertices such that $\delta^{0}(G) \geqslant(1 / 2-c) n$. If $\mathcal{P}$ is a non-trivial $\eta$-partition of $V(G)$ and $L_{\mathcal{P}}^{\mu}(H(G))$ is 2-transferral-free, then there exists $A \in \mathcal{P}$ such that $\operatorname{cyc}(A, A, \bar{A}) \leqslant \alpha n^{3}$.

Lemma 14. Suppose that $1 / n \ll c \ll \mu, \alpha \ll \gamma, \eta<1$ and $G$ is an oriented graph on $n$ vertices such that $\delta^{0}(G) \geqslant(1 / 2-c) n$. If $\mathcal{P}$ is a non-trivial $\eta$-partition of $V(G)$, $L_{\mathcal{P}}^{\mu}(H(G))$ is 2-transferral-free, and there exists $A \in \mathcal{P}$ such that $\operatorname{cyc}(A, A, \bar{A}) \leqslant \alpha n^{3}$, then $G$ is $\gamma$-extremal.

We prove Lemmas 11 and 12 in Section 2.2, Lemma 13 in Section 2.3, and Lemma 14 in Section 2.4.

Proof of Lemma 10. Introduce additional constants, $\beta^{\prime}, \mu$ and $\alpha$ so that

$$
1 / n \ll c \ll \beta \ll \beta^{\prime} \ll \mu \ll \alpha \ll \gamma<1 \text {. }
$$

By Lemma 12, with $\beta^{\prime}$ playing the role of $\beta$, for some $d^{\prime} \leqslant 4$, there exists $\mathcal{P}^{\prime}=\left\{V_{1}^{\prime}, \ldots, V_{d^{\prime}}^{\prime}\right\}$ a $\left(H(G), \beta^{\prime}, 8\right)$-closed 0.1-partition of $V(G)$. If $L_{\mathcal{P}^{\prime}}^{\mu}(H(G))$ contains a 2-transferral $\mathbf{u}_{i}-\mathbf{u}_{j}$ for distinct $i$ and $j$ in $\left[d^{\prime}\right]$, then merge the parts that correspond to the 2-transferral, i.e., consider the new partition $\mathcal{P}^{\prime}-V_{i}^{\prime}-V_{j}^{\prime}+\left(V_{i}^{\prime} \cup V_{j}^{\prime}\right)$. Continue to merge the parts that correspond to 2-transferrals until we have a partition $\mathcal{P}$ such that $L_{\mathcal{P}}^{\mu}(H(G))$ is 2-transferral-free. By Lemma 11, we can assume that $\mathcal{P}$ is an $(H(G), \beta, \ell)$-closed 0.1partition of $V(G)$ for some $\ell \leqslant 5^{3} \cdot 8=1000$. If $|\mathcal{P}|=1$, then $V(G)$ is $(H(G), \beta, \ell)$-closed which contradicts our assumptions, so we can assume that $\mathcal{P}$ is non-trivial. By Lemma 13 , there exists $A \in \mathcal{P}$ such that $\operatorname{cyc}(A, A, \bar{A}) \leqslant \alpha n^{3}$ and by Lemma 14 we have that $G$ is $\gamma$-extremal.

\subsection{Proofs of Lemmas 11 and 12}

We start this section with a proof of Lemma 11.

Proof of Lemma 11. Let $\xi$ be such that $\beta \ll \xi \ll \beta^{\prime} \ll 1 / \ell$. Note that distinct vertices $u_{0}$ and $v_{0}$ are $(H(G), \beta, 4 \ell+1)$-reachable if there exist at least $\xi n^{12 \ell+2}$ ordered $(12 \ell+2)$-tuples $\mathcal{T}$ that each can be permuted to form $(12 \ell+2)$-tuples $\left(u_{1}, \ldots, u_{12 \ell+2}\right)$ and $\left(v_{1}, \ldots, v_{12 \ell+2}\right)$ such that $u_{3 j} u_{3 j+1} u_{3 j+2}$ and $v_{3 j} v_{3 j+1} v_{3 j+2}$ are both cyclic triangles for every $0 \leqslant j \leqslant 4 \ell$. 
This is because $\beta<\xi^{2} / 2$; and there are only at most $(12 \ell+2)$ ! $<1 / \xi$ possible orderings for every such tuple; and only at most

$$
2(12 \ell+2) n^{12 \ell+1}+n \cdot(12 \ell+2)^{2} \cdot n^{12 \ell}<\xi n^{12 \ell+2} / 2
$$

such $(12 \ell+2)$-tuples that contain $u_{0}$ or $v_{0}$ or that have repeated vertices.

We will first show that if $u, v \in V(G)$ are $\left(H(G), \beta^{\prime}, \ell\right)$-reachable, then $u$ and $v$ are also $(H(G), \beta, 4 \ell+1)$-reachable. In particular, this will imply that, because $\mathcal{P}$ is $\left(H(G), \beta^{\prime}, \ell\right)$-closed, $\mathcal{P}$ is also $(H(G), \beta, 4 \ell+1)$-closed. Let $\mathcal{T}$ be the set of $(12 \ell+2)$ tuples $\left(x_{1}, x_{2}, \ldots, x_{12 \ell+2}\right)$ such that

- $\left\{x_{1}, x_{2}, \ldots, x_{3 \ell-1}\right\}$ is a $(H(G), u, v)$-linking $(3 \ell-1)$-set, and

- $G\left[\left\{x_{3 j}, x_{3 j+1}, x_{3 j+2}\right\}\right]$ is a cyclic triangle for $\ell \leqslant j \leqslant 4 \ell$.

Since $u$ and $v$ are $\left(H(G), \beta^{\prime}, \ell\right)$-reachable, there are at least $\beta^{\prime} n^{3 \ell-1}$ ways to select the first $3 \ell-1$ entries of a tuple in $\mathcal{T}$. For every such selection, there are exactly $(6 \operatorname{cyc}(V(G)))^{3 \ell+1}$ ways to select the remaining $9 \ell+3$ entries of a tuple in $\mathcal{T}$. To see that $6 \operatorname{cyc}(V(G)) \geqslant$ $2\left(\beta^{\prime}\right)^{2} n^{3}$, let $V_{q}$ be the part in $\mathcal{P}$ of largest cardinality. We have that $\left|V_{q}\right| \geqslant n / d \geqslant \beta^{\prime} n$. Because $V_{q}$ is $\left(H(G), \beta^{\prime}, \ell\right)$-closed, for every $v \in V_{q}$, the set $\tilde{N}_{H(G)}\left(\beta^{\prime}, \ell, v\right)$ is not empty, which implies that $v$ is in at least $\beta^{\prime} n^{2}$ cyclic triangles. Therefore, because $6 \operatorname{cyc}(V(G))$ is the number of ordered triples $(x, y, z)$ such that $G[\{x, y, z\}]$ is a cyclic triangle in $G$, we have that $6 \operatorname{cyc}(V(G)) \geqslant 2\left(\beta^{\prime}\right)^{2} n^{3}$. Hence,

$$
|\mathcal{T}| \geqslant \beta^{\prime} n^{3 \ell-1} \cdot\left(2\left(\beta^{\prime}\right)^{2} n^{3}\right)^{3 \ell+1}>\xi n^{12 \ell+2},
$$

so $u$ and $v$ are $(H(G), \beta, 4 \ell+1)$-reachable.

Now we will complete the proof by showing that if $u_{0} \in V_{i}$ and $v_{3} \in V_{j}$, then $u_{0}$ and $v_{3}$ are $(H(G), \beta, 4 \ell+1)$-reachable. By assumption, there are $A, B \in \mathcal{P}$ such that $\operatorname{cyc}\left(V_{i}, A, B\right)$ and $\operatorname{cyc}\left(A, B, V_{j}\right)$ are both at least $\mu n^{3}$. Let $\mathcal{T}$ be the set of $(12 \ell+2)$-tuples

$$
\left(v_{0}, v_{1}, v_{2}, u_{1}, u_{2}, u_{3}, w_{1}, \ldots, w_{12 \ell-4}\right)
$$

that satisfy the following:

- $v_{0}, v_{1}, v_{2}$ is a cyclic triangle with $v_{0} \in V_{i}, v_{1} \in A$ and $v_{2} \in B$;

- $u_{1}, u_{2}, u_{3}$ is a cyclic triangle with $u_{1} \in A$ and $u_{2} \in B$, and $u_{3} \in V_{j}$; and

- $\left\{w_{i(3 \ell-1)+1}, \ldots, w_{(i+1)(3 \ell-1)}\right\}$ is a $\left(H(G), u_{i}, v_{i}\right)$-linking $(3 \ell-1)$-set for $i \in\{0,1,2,3\}$.

Since $\operatorname{cyc}\left(V_{i}, A, B\right)$ and $\operatorname{cyc}\left(A, B, V_{j}\right)$ are both at least $\mu n^{3}$ and $V_{i}, V_{j}, A, B$ are all $\left(H(G), \beta^{\prime}, \ell\right)$-closed, we have that

$$
|\mathcal{T}| \geqslant\left(\mu n^{3}\right) \cdot\left(\mu n^{3}\right) \cdot\left(\beta^{\prime} n^{3 \ell-1}\right)^{4} \geqslant \xi n^{12 \ell+2},
$$

so $u_{0}$ and $v_{3}$ are $(H(G), \beta, 4 \ell+1)$-reachable.

To prove Lemma 12, we use the following lemma of Han [8]. 
Lemma 15 (Lemma 3.8 in [8]). Given $1 / n \ll \beta \ll \alpha \ll \delta, \delta^{\prime}, 1 / k$ the following holds. Let $H$ be a k-uniform hypergraph on $n$ vertices. Assume that $\left|\tilde{N}_{H}(\alpha, 1, v)\right| \geqslant \delta^{\prime} n$ for every $v \in V(H)$ and $\delta_{1}(H) \geqslant \delta\left(\begin{array}{l}n-1 \\ k-1\end{array}\right)$. Then for $d \leqslant \min \left\{\lfloor 1 / \delta\rfloor,\left\lfloor 1 / \delta^{\prime}\right\rfloor\right\}$ there exists an $\left(H, \beta, 2^{\lfloor 1 / \delta\rfloor-1}\right)$-closed partition $\mathcal{P}=\left\{V_{1}, \ldots, V_{d}\right\}$ of $V(H)$ such that for every $i \in$ $\{1, \ldots, d\}$ we have that $\left|V_{i}\right| \geqslant\left(\delta^{\prime}-\alpha\right) n$.

To apply Lemma 15 in our context, we need lower bounds on $\delta_{1}(H(G))$ and on $\left|\tilde{N}_{H(G)}(\alpha, 1, v)\right|$ for every vertex $v \in V(G)$ when $G$ is an oriented graph with sufficiently high minimum semidegree. The following series of lemmas provide these lower bounds. Note that Lemmas 16, 17, and 18 are also used in other sections.

Lemma 16. Suppose that $c>0$ and $G=(V, E)$ is an oriented graph on $n$ vertices such that $\delta^{0}(G) \geqslant(1 / 2-c) n$. Then, for every $A \subseteq V$,

$$
e^{+}(A, \bar{A}), e^{-}(A, \bar{A})=\frac{|A| \cdot|\bar{A}|}{2} \pm c|A| n .
$$

Proof. We first get a lower bound for $e^{+}(A, \bar{A})$ as follows,

$$
\begin{aligned}
e^{+}(A, \bar{A})=\sum_{x \in A} d^{+}(x)-d^{+}(x, A) \geqslant \\
\quad\left(\sum_{x \in A} d^{+}(x)\right)-\left(\begin{array}{c}
|A| \\
2
\end{array}\right) \geqslant|A|\left(\frac{1}{2}-c\right) n-\frac{|A|^{2}}{2}=\frac{|A| \cdot|\bar{A}|}{2}-c|A| n .
\end{aligned}
$$

By a similar computation, we have that $e^{-}(A, \bar{A}) \geqslant|A||\bar{A}| / 2-c|A| n$. The fact that

$$
e^{+}(A, \bar{A})+e^{-}(A, \bar{A}) \leqslant|A| \cdot|\bar{A}|,
$$

then implies the upper bounds.

The following lemma appears in [13]. We provide a proof for completeness.

Lemma 17. Suppose that $c>0$ and $G=(V, E)$ is an oriented graph on $n$ vertices such that $\delta^{0}(G) \geqslant(1 / 2-c) n$. Then, $\delta_{1}(H(G))=n^{2} / 8 \pm 2 c n^{2}$.

Proof. Let $u \in V$. We will show that $\operatorname{cyc}(u, V, V)=n^{2} / 8 \pm 2 c n^{2}$. Let $m=d^{+}(u)$ and assume $d^{+}(u) \leqslant d^{-}(u)$, so $m \leqslant(n-1) / 2$. By the minimum semidegree condition, $|\overline{N(u)}| \leqslant 2 c n$, so

$$
e^{+}\left(N^{+}(u), \overline{N(u)}\right) \leqslant m \cdot 2 c n .
$$

Since we can assume $c<1 / 2$,

$$
n^{2} / 4-c n^{2}<n^{2} / 4-c^{2} n^{2} \leqslant m(n-m) \leqslant n^{2} / 4 .
$$

With Lemma 16 and the fact that $m \leqslant n / 2$, we can compute that $\operatorname{cyc}(u, V, V)=$ $e^{+}\left(N^{+}(u), N^{-}(u)\right)$ is equal to

$$
e^{+}\left(N^{+}(u), \overline{N^{+}(u)}\right)-e^{+}\left(N^{+}(u), \overline{N(u)}\right)=\frac{m(n-m)}{2} \pm 3 c n m=n^{2} / 8 \pm 2 c n^{2} .
$$

Applying a similar argument when $d^{-}(u) \leqslant d^{+}(u)$ proves the lemma. 
Lemma 18. Suppose that $c>0$ and $G=(V, E)$ is an oriented graph on $n$ vertices such that $\delta^{0}(G) \geqslant(1 / 2-c) n$. Then, for every edge $u v \in E$,

$$
d^{-,+}(u v)-d^{+,-}(u v)=0 \pm 4 c n .
$$

This further implies that, for every pair of disjoint subsets $A$ and $B$ of $V$,

$$
\sum_{u v \in E^{+}(A, B)} \operatorname{cyc}(u, v, V) \geqslant \frac{e^{+}(A, B)^{2}}{2|A|}-c n^{3} .
$$

Proof. To prove the first part of the lemma, note that

$$
d^{+,-}(x y)=d^{+}(x)-d^{+,+}(x y)-\left|N^{+}(x) \backslash N(y)\right|,
$$

and similarly $d^{-,+}(x y)=d^{+}(y)-d^{+,+}(x y)-\left|N^{+}(y) \backslash N(x)\right|$. Therefore,

$$
d^{-,+}(x y)-d^{+,-}(x y)=\left(d^{+}(x)-d^{+}(y)\right)+\left(\left|N^{+}(y) \backslash N(x)\right|-\left|N^{+}(x) \backslash N(y)\right|\right)=0 \pm 4 c n,
$$

by the minimum semidegree condition.

To prove the second part of the lemma, note that if $u \in A$ and $v \in N^{+}(u, B)$, then

$$
d^{+,-}(u v) \geqslant d^{-}\left(v, N^{+}(u, B)\right) .
$$

This implies that

$$
\begin{aligned}
\sum_{v \in N^{+}(u, B)} d^{+,-}(u v) \geqslant & \sum_{v \in N^{+}(u, B)} d^{-}\left(v, N^{+}(u, B)\right)= \\
& \quad e\left(N^{+}(u, B)\right) \geqslant d^{+}(u, B)\left(\frac{d^{+}(u, B)-2 c n}{2}\right),
\end{aligned}
$$

where the last inequality follows from the fact that $n-2 \delta^{0}(G) \leqslant 2 c n$. This observation, with the first part of the lemma gives us that

$$
\begin{aligned}
\sum_{v \in N^{+}(u, B)} \operatorname{cyc}(u, v, V)= & \sum_{v \in N^{+}(u, B)} d^{-,+}(u v) \geqslant \\
& \sum_{v \in N^{+}(u, B)}\left(d^{+,-}(u v)-4 c n\right) \geqslant d^{+}(u, B)\left(\frac{d^{+}(u, B)}{2}-5 c n\right) .
\end{aligned}
$$

Letting $m=e^{+}(A, B)$ and $|A|=a$, we have that, by the convexity of $f(x)=x^{2}$,

$\sum_{u v \in E^{+}(A, B)} \operatorname{cyc}(u, v, V) \geqslant \sum_{u \in A} d^{+}(u, B)\left(\frac{d^{+}(u, B)}{2}-5 c n\right) \geqslant \frac{a}{2}\left(\frac{m}{a}\right)^{2}-m \cdot 5 c n \geqslant \frac{m^{2}}{2 a}-c n^{3}$,

where the last inequality follows because, by Lemma $16, m \leqslant n^{2} / 5$. 
Lemma 19. Suppose $1 / n \ll c \ll \alpha \ll 1$ and that $G=(V, E)$ is an oriented graph on $n$ vertices such that $\delta^{0}(G) \geqslant(1 / 2-c) n$. Then, for every $v \in V$,

$$
\left|\tilde{N}_{H(G)}(\alpha, 1, v)\right| \geqslant\left(\frac{1}{8}-10 \alpha\right) n .
$$

Proof. Fix $v \in V$ and let $N=\tilde{N}_{H(G)}(\alpha, 1, v)$ and $\bar{N}=V \backslash N$. For every $U \subseteq V$, let $T(U)$ be the set of ordered triples $(x, y, u)$ such that $u \in U, x y \in E$ and both $v x y$ and $x y u$ are cyclic triangles. By definition, $u \in \bar{N}$ if and only if $|T(\{u\})|<\alpha n^{2}$, so

$$
|T(N)|=|T(V)|-|T(\bar{N})| \geqslant|T(V)|-\alpha n^{3} .
$$

For every $u \in V$, Lemma 17 implies that $|T(\{u\})| \leqslant(1 / 8+2 c) n^{2}$, so

$$
(1 / 8+2 c) n^{2} \cdot|N| \geqslant|T(N)| \geqslant|T(V)|-\alpha n^{3}
$$

Therefore, to show that $|N| \geqslant(1 / 8-10 \alpha) n$ and complete the proof, it suffices to prove that $|T(V)| \geqslant(1 / 64-2 c) n^{3}$.

Let $m=e^{+}\left(N^{+}(v), N^{-}(v)\right)$. By Lemma 18, we have that

$$
|T(V)|=\sum_{x y \in E^{+}\left(N^{+}(v), N^{-}(v)\right)} \operatorname{cyc}(x, y, V) \geqslant \frac{m^{2}}{2 d^{+}(v)}-c n^{3} .
$$

Because Lemma 17 implies that $m \geqslant(1 / 8-2 c) n^{2}$, we have that

$$
\frac{m^{2}}{2 d^{+}(v)} \geqslant \frac{m n}{2} \cdot \frac{1 / 8-2 c}{1 / 2+c} \geqslant(1 / 16-c) n^{3} \cdot(1 / 4-5 c) \geqslant(1 / 64-c) n^{3},
$$

which, with (2), completes the proof of the lemma.

Proof of Lemma 12. Let $\delta=2 / 9, \delta^{\prime}=1 / 8-1 / 100$ and let $\beta \ll \alpha \ll 1$. By Lemmas 17 and 19 , we have that $\delta_{1}(H(G)) \geqslant(1 / 8-2 c) n^{2}>\delta\left(\begin{array}{c}n-1 \\ 2\end{array}\right)$ and that $\left|\tilde{N}_{H(G)}(\alpha, 1, v)\right| \geqslant$ $(1 / 8-10 \alpha) n \geqslant \delta^{\prime} n$ for every $v \in V(G)$. Since $\min \left\{\lfloor 1 / \delta\rfloor,\left\lfloor 1 / \delta^{\prime}\right\rfloor\right\}=4$, Lemma 15 implies that, for some $d \leqslant 4$, there exists $\mathcal{P}=\left\{V_{1}, \ldots, V_{d}\right\}$ a $(H(G), \beta, 8)$-closed partition of $V(G)$ such that and $\left|V_{i}\right| \geqslant\left(\delta^{\prime}-\alpha\right) n>n / 9$ for every $i \in[d]$.

\subsection{Proof of Lemma 13}

In this section, we prove Lemma 13. In an effort to explain the structure of the proof at a high level, we first informally discuss how to derive a contradiction in the following situation. Suppose that $G$ is a regular tournament on $n$ vertices that has an $\eta$-partition $\mathcal{P}$ of $V(G)$ with three distinct parts $A, B, D \in \mathcal{P}$ such that

(S1) $A$ is the largest part in $\mathcal{P}$,

(S2) $G[A], G[B]$ are transitive tournaments,

(S3) every cyclic triangle with at least two vertices in $A$ has one vertex in $B$, and 
(S4) every cyclic triangle with at least two vertices in $B$ has one vertex in $D$.

These conditions are an idealized version of the conditions we will use to produce a contradiction to prove Lemma 13. (Note that (S3) and (S4) together imply (S2).)

Let $C=\overline{A \cup B}$. Since $G[A]$ is a transitive tournament, there exist $x^{+}, x^{-} \in A$ such that $d^{+}\left(x^{+}, A\right)=d^{-}\left(x^{-}, A\right)=|A|-1$. This implies that if we let $C^{-}=N^{-}\left(x^{+}, C\right)$, then we have that $\left|C^{-}\right|$is at least

$$
\delta^{0}(G)-|B|=(n-1) / 2-(|A|+|B|) / 2+(|A|-|B|) / 2=|C| / 2+(|A|-|B|) / 2-1 / 2 .
$$

The same lower bound holds for $\left|C^{+}\right|$when we let $C^{+}=N^{+}\left(x^{-}, C\right)$. Let $v \in C^{-}$. Note that, because no cyclic triangle contains both $v$ and $x^{+}$and has its third vertex in $A$, we have that $N^{+}(v, A)=A$. Similarly $N^{-}(v, A)=A$ for every $v \in C^{+}$. Therefore, $C^{+}$ and $C^{-}$are disjoint, so, with (3) and the fact that $|A| \geqslant|B|$, we have that $\left|C^{+}\right|,\left|C^{-}\right|=$ $|C| / 2 \pm 1 / 2$ and $|B| \geqslant|A|-1$.

Since $|B| \geqslant|A|-1$ and $A$ is the largest part in $\mathcal{P}$, we have that $|D| \leqslant|A| \leqslant|B|+1$. Therefore, using the same logic with $B$ and $D$ playing the roles of $A$ and $C$, respectively, we can find $y^{+}, y^{-} \in B$ such that $d^{+}\left(y^{+}, B\right)=d^{-}\left(y^{-}, B\right)=|B|-1$ and the disjoint union of $N^{-}\left(y^{+}, \overline{B \cup D}\right)$ and $N^{+}\left(y^{-}, \overline{B \cup D}\right)$ covers all but at most two vertices of $\overline{B \cup D}$. In particular, because $A \subseteq \overline{B \cup D}$, if we let $A^{-}=N^{-}\left(y^{+}, A\right)$ and $A^{+}=N^{+}\left(y^{-}, A\right)$ we have that $A^{-}$and $A^{+}$are disjoint sets and $\left|A^{-} \cup A^{+}\right| \geqslant|A|-2$. Assume that $\left|A^{-}\right| \geqslant\left|A^{+}\right|$, so $\left|A^{-}\right| \geqslant|A| / 2-1$. (Similar logic gives a contradiction when $\left|A^{+}\right| \geqslant\left|A^{-}\right|$). Because $G\left[A^{-}\right]$ is a transitive tournament, there exists $x \in A^{-}$such that $d^{+}\left(x, A^{-}\right)=\left|A^{-}\right|-1$.

To get a contradiction, we now argue that the out-degree of $x$ is greater than $(n-1) / 2$. Recall that $N^{-}(v, A)=A$ for every $v \in C^{+}$. This means that $N^{+}(x)$ contains $C^{+}$. Moreover, since $x y^{+}$is an edge and there are no triangles with one vertex in $A$ and two vertices in $B$, we have that $N^{+}(x)$ contains $B$. Therefore, $d^{+}(x) \geqslant\left|A^{-} \backslash x\right|+|B|+\left|C^{+}\right|$. Recall that $\left|A^{-}\right| \geqslant|A| / 2-1$ and $\left|C^{+}\right| \geqslant|C| / 2-1 / 2$. Therefore, the fact that $\{A, B, C\}$ partitions $V(G)$ implies that $d^{+}(x) \geqslant n / 2+|B| / 2-5 / 2$. Because $\mathcal{P}$ is an $\eta$-partition, we have that $|B| \geqslant \eta n$, so this contradicts the fact that $G$ is a regular tournament. (See Figure 1.)

Lemma 20. Suppose that $1 / n \ll \alpha, c \ll \xi, \beta \ll 1$ and that $G$ is an oriented graph on $n$ vertices such that $\delta^{0}(G) \geqslant(1 / 2-c) n$. If $A, B \subseteq V(G)$ are disjoint and $e^{+}(A, B) \leqslant \alpha n^{2}$, then $\left|\mathrm{SN}_{\beta}^{-}(A) \cap B\right| \geqslant|B|-\xi n$ and $\left|\mathrm{SN}_{\beta}^{+}(B) \cap A\right| \geqslant|A|-\xi n$

Proof. Note that for every $v \in B \backslash \mathrm{SN}_{\beta}^{-}(A)$, we have that $d^{-}(v, A) \geqslant \beta n-\left(n-2 \delta^{0}(G)\right) \geqslant$ $\beta n / 2$, and also, for every $v \in A \backslash \mathrm{SN}_{\beta}^{+}(B)$, we have that $d^{+}(v, B) \geqslant \beta n / 2$. Therefore, if we let $m$ be the maximum of $\left|B \backslash \mathrm{SN}_{\beta}^{-}(A)\right|$ and $\left|A \backslash \mathrm{SN}_{\beta}^{+}(B)\right|$, then $m \cdot \beta n / 2 \leqslant e^{+}(A, B) \leqslant \alpha n^{2}$, which implies that $m \leqslant \xi n$.

Lemma 21. Suppose that $1 / n \ll c, \alpha \ll \beta<1$, and that $G=(V, E)$ is an $n$-vertex oriented graph such that $\delta^{0}(G) \geqslant(1 / 2-c) n$. If $A \subseteq V$ and $\operatorname{cyc}(A) \leqslant \alpha n^{3}$, then, for $\sigma \in\{+,-\}$, there exists $x^{\sigma} \in A$ such that $d^{\sigma}\left(x^{\sigma}, A\right) \geqslant|A|-\beta n$. 


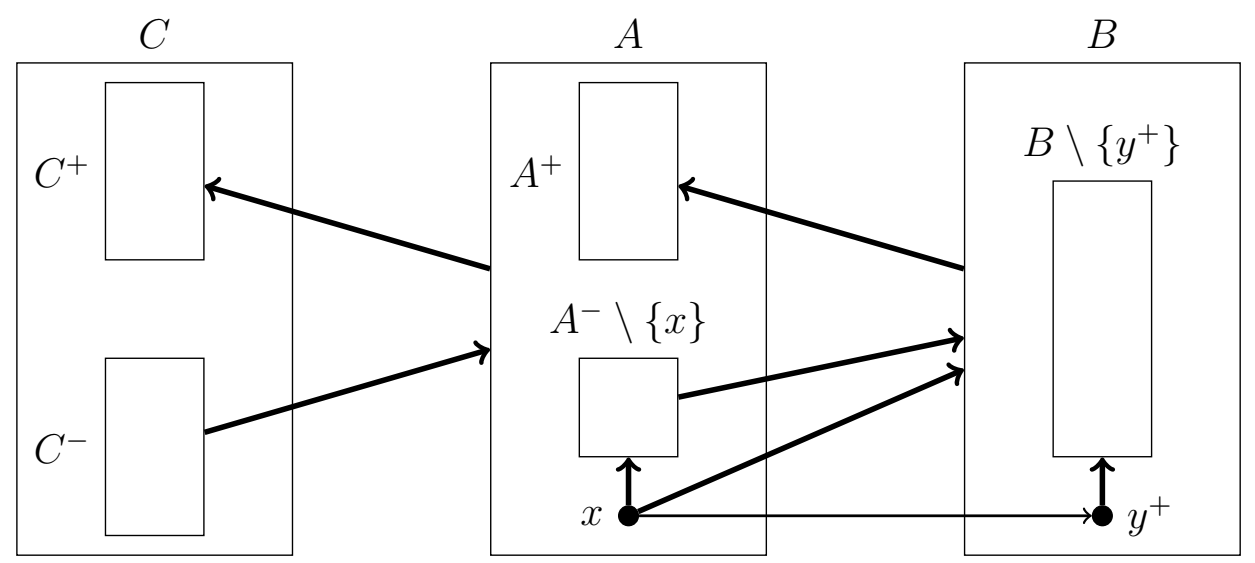

Figure 1: The situation at the end of the sketch of the proof of Lemma 13. The outneighborhood of the vertex $x$ contains $A^{-} \backslash\{x\} \cup C^{+} \cup B$ which has cardinality larger than $(n-1) / 2$, a contradiction to the assumption that $G$ is a regular tournament.

Proof. Pick $\xi$ so that

$$
1 / n \ll c, \alpha \ll \xi \ll \beta \ll 1 .
$$

Let $X$ be the set of vertices $x$ in $A$ such that $\operatorname{cyc}(x, A, A) \leqslant \alpha^{1 / 2} n^{2}$. Since

$$
(|A|-|X|) \cdot \alpha^{1 / 2} n^{2} \leqslant 3 \operatorname{cyc}(A) \leqslant 3 \alpha n^{3}
$$

we have that

$$
|X| \geqslant|A|-3 \alpha^{1 / 2} n \text {. }
$$

Pick $x^{+} \in X$ so as to maximize $d^{+}\left(x^{+}, X\right)$, and let $X^{+}=N^{+}\left(x^{+}, X\right)$ and $X^{-}=$ $N^{-}\left(x^{+}, X\right)$. Note that, because of the minimum semidegree condition, $\left|X^{-}\right| \geqslant|X|-$ $\left|X^{+}\right|-2 c n$, and that $\alpha^{1 / 2} n^{2} \geqslant \operatorname{cyc}\left(x^{+}, A, A\right) \geqslant e^{+}\left(X^{+}, X^{-}\right)$. Therefore, with Lemma 20, we have that

$$
\left|\mathrm{SN}_{\xi}^{-}\left(X^{+}\right) \cap X^{-}\right| \geqslant\left|X^{-}\right|-\xi n \geqslant|X|-\left|X^{+}\right|-2 c n-\xi n,
$$

and, with the minimum semidegree condition, there exists $y \in \mathrm{SN}_{\xi}^{-}\left(X^{+}\right) \cap X^{-}$such that

$$
d^{+}\left(y, X^{-}\right) \geqslant\left(\left|\mathrm{SN}_{\xi}^{-}\left(X^{+}\right) \cap X^{-}\right|-2 c n\right) / 2 \geqslant\left(|X|-\left|X^{+}\right|-2 \xi n\right) / 2
$$

Because $y \in \mathrm{SN}_{\xi}^{-}\left(X^{+}\right)$, we have that

$$
d^{+}\left(y, X^{+}\right) \geqslant\left|X^{+}\right|-\xi n .
$$

Therefore, by the selection of $x^{+}$we have that,

$\left|X^{+}\right| \geqslant d^{+}\left(y, X^{+}\right)+d^{+}\left(y, X^{-}\right) \geqslant\left(\left|X^{+}\right|-\xi n\right)+\left(|X|-\left|X^{+}\right|-2 \xi n\right) / 2=\left(|X|+\left|X^{+}\right|-4 \xi n\right) / 2$

which, with (4), implies $d^{+}\left(x^{+}, A\right) \geqslant\left|X^{+}\right| \geqslant|X|-4 \xi n \geqslant|A|-\beta n$. By a similar argument, we can find $x^{-} \in A$, such that $d^{-}\left(x^{-}, A\right) \geqslant|A|-\beta n$. 
Lemma 22. Let $1 / n \ll \alpha, c \ll \xi \ll \eta<1$. Let $G=(V, E)$ be an $n$-vertex oriented graph such that $\delta^{0}(G) \geqslant(1 / 2-c) n$. For every partition $\{A, B, C\}$ of $V$ such that $|A| \geqslant \eta n$ and $\operatorname{cyc}(A), \operatorname{cyc}(A, A, C) \leqslant \alpha n^{3}$ there exist disjoint subsets $C^{+}$and $C^{-}$of $C$ such that, for $\sigma \in\{+,-\}$,

$$
e^{-\sigma}\left(A, C^{\sigma}\right) \leqslant \xi n^{2} \quad \text { and } \quad\left|C^{\sigma}\right| \geqslant|C| / 2+(|A|-|B|) / 2-\xi n .
$$

Proof. We will prove the lemma by showing that, with $\xi / 2$ playing the role of $\xi$, there exist subsets $C^{+}$and $C^{-}$of $C$ that meet the conditions of the lemma except that $C^{+} \cap C^{-}$ might not be empty. This will prove the lemma, because then, by the minimum semidegree condition,

$$
(|A|-2 c n)\left|C^{+} \cap C^{-}\right| \leqslant e^{+}\left(A, C^{+} \cap C^{-}\right)+e^{-}\left(A, C^{+} \cap C^{-}\right) \leqslant \xi n^{2},
$$

and this, with the fact that $|A| \geqslant \eta n$, implies that $\left|C^{+} \cap C^{-}\right| \leqslant \xi n / 2$, which means that the sets $C^{+} \backslash C^{-}$and $C^{-} \backslash C^{+}$are disjoint sets that meet the conditions of the lemma. Furthermore, we will only prove that such a $C^{+}$exists, because the existence of the desired set $C^{-}$follows by a similar argument.

Let $X$ be the set of vertices $x$ in $A$ such that $\operatorname{cyc}(x, A, C) \leqslant \alpha^{1 / 2} n^{2}$. Because

$$
(|A|-|X|) \cdot \alpha^{1 / 2} n^{2} \leqslant 3 \operatorname{cyc}(A, A, C) \leqslant 3 \alpha n^{3}
$$

we have that $|X| \geqslant|A|-3 \alpha^{1 / 2} n$. Since $\operatorname{cyc}(X, X, X) \leqslant \operatorname{cyc}(A, A, A) \leqslant \alpha n^{3}$, Lemma 21 implies that there exists $x \in X$ such that

$$
d^{-}(x, A) \geqslant d^{-}(x, X) \geqslant|X|-\xi n / 4 \geqslant|A|-\xi n / 3 .
$$

Let $C^{+}=N^{+}(x, C)$. Because every edge directed from $C^{+}$to $N^{-}(x, A)$ corresponds to a triangle in $\operatorname{cyc}(x, A, C)$, we have that

$$
e^{-}\left(A, C^{+}\right) \leqslant \operatorname{cyc}(x, A, C)+e^{-}\left(A \backslash N^{-}(x, A), C^{+}\right) \leqslant \xi n^{2} / 2 .
$$

We also have that

$\left|C^{+}\right| \geqslant \delta^{0}(G)-d^{+}(x, A)-|B| \geqslant n / 2-|B|-c n-\xi n / 3 \geqslant|C| / 2+(|A|-|B|) / 2-\xi n / 2$.

This completes the proof of the lemma.

Proof of Lemma 13. Pick $\beta, \alpha^{\prime}$ and $\xi$ so that

$$
1 / n \ll c \ll \mu \ll \alpha^{\prime} \ll \alpha, \xi \ll \beta \ll \eta<1 .
$$

For a contradiction, assume that

$$
\operatorname{cyc}\left(V_{i}, V_{i}, \overline{V_{i}}\right) \geqslant \alpha n^{3} \quad \text { for all } V_{i} \in \mathcal{P}=\left\{V_{1}, \ldots, V_{d}\right\} .
$$

Because $\mathcal{P}$ is an $\eta$-partition we have that $1 / d \geqslant \eta$ which implies that

$$
\forall J \subseteq[d], \forall i \in[d], \exists j^{\prime} \in J \text { such that } \operatorname{cyc}\left(V_{i}, V_{i}, V_{j^{\prime}}\right) \geqslant \eta \cdot c\left(V_{i}, V_{i}, \bigcup_{j \in J} V_{j}\right) .
$$


Let $A=V_{x}$ be the largest part in $\mathcal{P}$. By (5) and (6) with $J=[d]-x$, there exists $V_{y}=B \in \mathcal{P}-A$ such that $\operatorname{cyc}(A, A, B) \geqslant \alpha^{\prime} n^{3}$. Let $C=V \backslash(A \cup B)$. By (5) and (6) with $J=[d]-y$, we also have $V_{z}=D \in \mathcal{P}-B$ such that $c(B, B, D) \geqslant \alpha^{\prime} n^{3}$. Let $F=V \backslash(B \cup D)$.

Note that $\mathbf{v}_{1}=2 \mathbf{u}_{x}+\mathbf{u}_{y} \in I_{\mathcal{P}}^{\mu}(H(G))$ and $\mathbf{v}_{2}=2 \mathbf{u}_{y}+\mathbf{u}_{z} \in I_{\mathcal{P}}^{\mu}(H(G))$. If $A=D$, then $\mathbf{v}_{1}-\mathbf{v}_{2}$ is a 2-transferral in $L_{\mathcal{P}}^{\mu}(H(G))$. Furthermore, if cyc $(A) \geqslant \alpha^{\prime} n^{3}$, then $\mathbf{v}_{3}=3 \mathbf{u}_{x} \in$ $I_{\mathcal{P}}^{\mu}(H(G))$, so $\mathbf{v}_{1}-\mathbf{v}_{3}$ is a 2 -transferral in $L_{\mathcal{P}}^{\mu}(H(G))$. Also, if cyc $(A, A, C) \geqslant \alpha^{\prime} n^{3}$, then, by (6) with $J=[d]-x-y$, there exists $w \in[d]-x-y$ such that $\mathbf{v}_{4}=2 \mathbf{u}_{x}+\mathbf{u}_{w} \in I_{\mathcal{P}}^{\mu}(H(G))$, so $\mathbf{v}_{1}-\mathbf{v}_{4}$ is a 2-transferral in $L_{\mathcal{P}}^{\mu}(H(G))$. With additional similar arguments, the fact that there are no 2-transferrals in $L_{\mathcal{P}}^{\mu}(H(G))$ implies that $A \neq D$ and

$$
\operatorname{cyc}(A), \operatorname{cyc}(A, A, C), \operatorname{cyc}(B), \operatorname{cyc}(B, B, F)<\alpha^{\prime} n^{3} \text {. }
$$

By Lemma 22, there exist disjoint subsets $C^{+}, C^{-}$of $C$ such that, for $\sigma \in\{-,+\}$,

$$
e^{-\sigma}\left(A, C^{\sigma}\right) \leqslant \xi n^{2}
$$

and

$$
\left|C^{\sigma}\right| \geqslant|C| / 2+(|A|-|B|) / 2-\xi n .
$$

By the selection of $A$ we have that $|A| \geqslant|B|$, so (9) implies that

$$
\left|C^{\sigma}\right| \geqslant|C| / 2-\xi n \text {. }
$$

Because $C^{+}$and $C^{-}$are disjoint subsets of $C$, we have that $\min \left\{\left|C^{+}\right|,\left|C^{-}\right|\right\} \leqslant|C| / 2$, so, with (9),

$$
|B| \geqslant|A|-2 \xi n \text {. }
$$

By Lemma 22, with $B$ and $F$ playing the roles of $A$ and $C$, respectively, there are disjoint subsets $F^{+}$and $F^{-}$of $F$ such that, for $\sigma \in\{-,+\}$,

$$
e^{-\sigma}\left(B, F^{\sigma}\right) \leqslant \xi n^{2}
$$

and

$$
\left|F^{\sigma}\right| \geqslant|F| / 2+(|B|-|D|) / 2-\xi n .
$$

By the selection of $A$ and (11), we have that

$$
|B|+2 \xi n \geqslant|A| \geqslant|D|,
$$

so $\left|F^{\sigma}\right| \geqslant|F| / 2-2 \xi n$, and

$$
\left|F^{+}\right|+\left|F^{-}\right| \geqslant|F|-4 \xi n .
$$

Note that $A \subseteq F$ and fix $\sigma^{*} \in\{-,+\}$ so that $\left|F^{-\sigma^{*}} \cap A\right| \geqslant\left|F^{\sigma^{*}} \cap A\right|$. By (13) and the selection of $\sigma^{*}$, we have that

$$
\left|F^{-\sigma^{*}} \cap A\right| \geqslant\left(\left|F^{+} \cap A\right|+\left|F^{-} \cap A\right|\right) / 2 \geqslant(|A|-4 \xi n) / 2=|A| / 2-2 \xi n .
$$


Note that Lemma 20, with (8), implies that

$$
\left|A \backslash \mathrm{SN}_{\beta}^{\sigma^{*}}\left(C^{\sigma^{*}}\right)\right| \leqslant \beta n / 2,
$$

and Lemma 20, with (12) and (14), implies that

$$
\left|\mathrm{SN}_{\beta}^{\sigma^{*}}(B) \cap F^{-\sigma^{*}} \cap A\right| \geqslant|A| / 2-\beta n / 2 .
$$

Therefore, if we set $A^{\prime}=\operatorname{SN}_{\beta}^{\sigma^{*}}(B) \cap \operatorname{SN}_{\beta}^{\sigma^{*}}\left(C^{\sigma^{*}}\right) \cap A$, then

$$
\left|A^{\prime}\right| \geqslant\left|\mathrm{SN}_{\beta}^{\sigma^{*}}(B) \cap F^{-\sigma^{*}} \cap A\right|-\left|A \backslash \mathrm{SN}_{\beta}^{\sigma^{*}}\left(C^{\sigma^{*}}\right)\right| \geqslant|A| / 2-\beta n .
$$

Since $\operatorname{cyc}\left(A^{\prime}\right) \leqslant \operatorname{cyc}(A) \leqslant \alpha n^{3}$, Lemma 21 implies that there exists $x \in A^{\prime}$ such that

$$
d^{\sigma^{*}}\left(x, A^{\prime}\right) \geqslant\left|A^{\prime}\right|-\beta n \geqslant|A| / 2-2 \beta n .
$$

Recall that $x \in \mathrm{SN}_{\beta}^{\sigma^{*}}(B) \cap \mathrm{SN}_{\beta}^{\sigma^{*}}\left(C^{\sigma^{*}}\right)$. This implies that $d^{\sigma^{*}}(x, B) \geqslant|B|-\beta n$, and, with (10), we have that

$$
d^{\sigma^{*}}\left(x, C^{\sigma^{*}}\right) \geqslant\left|C^{\sigma^{*}}\right|-\beta n \geqslant|C| / 2-2 \beta n .
$$

Therefore, with the fact that $|B| \geqslant \eta n$, we have

$d^{\sigma^{*}}(x) \geqslant(|A| / 2-2 \beta n)+(|B|-\beta n)+(|C| / 2-2 \beta n)=n / 2+|B| / 2-5 \beta n>(1 / 2+c) n$,

so $d^{-\sigma^{*}}(x)<(1 / 2-c) n$, a contradiction to the minimum semidegree condition.

\subsection{Proof of Lemma 14}

We need the following lemma for the proof of Lemma 14. Informally, it says that, in an oriented graph $G$ with sufficiently high minimum semidegree, if $A$ is a reasonably large subset of $V(G)$ for which $\operatorname{cyc}(A, A, \bar{A})$ is $o\left(n^{3}\right)$, then essentially half of the vertices in $\bar{A}$ have almost all of $A$ as out-neighbors and half of the vertices in $\bar{A}$ have almost all of $A$ as in-neighbors. From this, we then argue that $|A|$ cannot be much larger than $n / 3$.

At a high-level, we use Lemma 23 to prove Lemma 14 in the following way (the actual proof of Lemma 14 appears after the proof of Lemma 23). We start with a non-trivial $\eta$-partition of $G$ such that $L_{\mathcal{P}}^{\mu}(H(G))$ is 2-transferral-free and such that there exists $A \in \mathcal{P}$ such that $\operatorname{cyc}(A, A, \bar{A})$ is $o\left(n^{3}\right)$. We then use Lemma 23 to get a partition $\left\{S^{+}, S^{-}\right\}$of $\bar{A}$ such that $S^{+}$and $S^{-}$have roughly the same size and such that $e^{+}\left(A, S^{-}\right)$and $e^{+}\left(S^{+}, A\right)$ are both $o\left(n^{2}\right)$. We can then finish the proof by showing that $A, S^{+}$and $S^{-}$each have roughly the same size (which is implied if $\left|S^{+}\right|$is not much more than $n / 3$ ) and that $e^{+}\left(S^{-}, S^{+}\right)$is $o\left(n^{2}\right)$. To this end, we use the fact that $L_{\mathcal{P}}^{\mu}(H(G))$ is 2-transferral-free to show that $\operatorname{cyc}\left(S^{+}, S^{+}, \overline{S^{+}}\right)$is $o\left(n^{3}\right)$, and then apply Lemma 23 again with $S^{+}$now playing the role of $A$.

Lemma 23. Suppose that

$$
1 / n \ll c \ll \alpha \ll \beta \ll \xi \ll \eta<1,
$$

and $G=(V, E)$ is an oriented graph such that $\delta^{0}(G) \geqslant(1 / 2-c) n$. For every $A \subseteq V$ such that $|A|>\eta n$ and $\operatorname{cyc}(A, A, \bar{A}) \leqslant \alpha n^{3}$, we have that 
- $\left|\mathrm{SN}_{\beta}^{+}(A)\right|,\left|\mathrm{SN}_{\beta}^{-}(A)\right|=|\bar{A}| / 2 \pm \xi n$, and

- $|A| \leqslant(1 / 3+\xi) n$.

Proof. Let $S^{+}=\mathrm{SN}_{\beta}^{+}(A), S^{-}=\mathrm{SN}_{\beta}^{-}(A)$ and $S=V \backslash\left(S^{+} \cup S^{-} \cup A\right)$, so $\left\{S, S^{+}, S^{-}, A\right\}$ is a partition of $V$. We will first prove that

$$
|S| \leqslant \beta n \text {. }
$$

To this end, let $x \in S \subseteq \bar{A}$ and then $d^{\sigma}(x, A) \leqslant|A|-\beta n$ for any $\sigma \in\{+,-\}$. Since $\delta^{0}(G) \geqslant(1 / 2-c) n$, then $x$ must have

$$
d^{\sigma}(x, A) \geqslant|A|-d^{-\sigma}(x, A)-2 c n \geqslant \beta n-2 c n \geqslant \beta n / 2 .
$$

Therefore,

$$
\begin{aligned}
|S|(\beta n)^{2} / 4 & \leqslant \sum_{x \in S} d^{+}(x, A) \cdot d^{-}(x, A) \leqslant \sum_{x \in \bar{A}} d^{+}(x, A) \cdot d^{-}(x, A) \\
& =\sum_{x y \in E(A)} d^{-,+}(x y, \bar{A})+d^{+,-}(x y, \bar{A})=\operatorname{cyc}(A, A, \bar{A})+\sum_{x y \in E(A)} d^{+,-}(x y, \bar{A}) .
\end{aligned}
$$

By Lemma 18, we have that

$$
\begin{aligned}
\sum_{x y \in E(A)} d^{+,-}(x y, \bar{A}) & =\sum_{x y \in E(A)}\left(d^{+,-}(x y)-d^{+,-}(x y, A)\right) \\
& \leqslant \sum_{x y \in E(A)}\left(d^{-,+}(x y)-d^{+,-}(x y, A)+4 c n\right) \\
& =\sum_{x y \in E(A)}\left(d^{-,+}(x y, \bar{A})+d^{-,+}(x y, A)-d^{+,-}(x y, A)+4 c n\right) \\
& =\operatorname{cyc}(A, A, \bar{A})+3 \operatorname{cyc}(A)-\operatorname{trn}(A)+4 c n \cdot e(A) .
\end{aligned}
$$

Because $e(A) /|A|=\frac{\sum_{v \in A} d^{+}(v, A)}{|A|}=\frac{\sum_{v \in A} d(v, A)}{2|A|} \geqslant \frac{|A|-2 c n}{2}$ and $f(x)=\left(\begin{array}{l}x \\ 2\end{array}\right)$ is convex, we have that

$$
\operatorname{trn}(A)=\sum_{v \in A}\left(\begin{array}{c}
d^{+}(v, A) \\
2
\end{array}\right) \geqslant|A|\left(\begin{array}{c}
e(A) /|A| \\
2
\end{array}\right) \geqslant \frac{|A|(|A|-2 c n-1)^{2}}{8} \geqslant \frac{|A|^{3}}{8}-c n^{3}
$$

Therefore, since $\operatorname{cyc}(A)+\operatorname{trn}(A) \leqslant\left(\begin{array}{c}|A| \\ 3\end{array}\right)$,

$$
3 \operatorname{cyc}(A)-\operatorname{trn}(A) \leqslant 3\left(\begin{array}{c}
|A| \\
3
\end{array}\right)-4 \operatorname{trn}(A) \leqslant 4 c n^{3}
$$

Combining (16), (17) and (18), we have that

$$
|S|(\beta n)^{2} / 4 \leqslant 2 \operatorname{cyc}(A, A, \bar{A})+8 c n^{3} \leqslant 3 \alpha n^{3},
$$


and (15) holds.

For $\sigma \in\{+,-\}$, we have that $e^{\sigma}(A, \bar{A}) \geqslant(|A|-\beta n) \cdot\left|S^{\sigma}\right|$ and, by (15) and the definition of $S^{-\sigma}$,

$$
e^{\sigma}(A, \bar{A}) \leqslant|A| \cdot\left|S^{\sigma}\right|+(|A|-\beta n) \cdot|S|+\beta n \cdot\left|S^{-\sigma}\right| \leqslant|A| \cdot\left|S^{\sigma}\right|+2 \beta n^{2},
$$

so $|A| \cdot\left|S^{\sigma}\right|=e^{\sigma}(A, \bar{A}) \pm 2 \beta n^{2}$. With Lemma 16 , we have that

$$
|A| \cdot\left|S^{+}\right|=e^{+}(A, \bar{A}) \pm 2 \beta n^{2}=e^{-}(A, \bar{A}) \pm 4 \beta n^{2}=|A| \cdot\left|S^{-}\right| \pm 6 \beta n^{2} .
$$

Therefore, because $|A| \geqslant \eta n$, we have that $\left|S^{+}\right|=\left|S^{-}\right| \pm \xi n$. This with (15) implies that

$$
|\bar{A}|=n-|A|=\left|S^{+}\right|+\left|S^{-}\right|+|S|=2\left|S^{\sigma}\right| \pm 2 \xi n,
$$

which proves the first part of the lemma.

We will prove the second part of the lemma by contradiction, so assume that $|A|>$ $(1 / 3+\xi) n$. There exists $x \in S^{+}$such that $d^{+}\left(x, S^{+}\right)<\left|S^{+}\right| / 2$. Using the fact that $|\bar{A}|<(2 / 3-\xi) n$, and, by the first part of the lemma, $\left|S^{+}\right| \geqslant|\bar{A}| / 2-\xi n$, we have that

$$
d^{+}(x, \bar{A})<|S|+\left|S^{-}\right|+\left|S^{+}\right| / 2=|\bar{A}|-\left|S^{+}\right| / 2 \leqslant|\bar{A}|-|\bar{A}| / 4+\xi n / 2<n / 2-\xi n / 4 .
$$

Because $x \in S^{+}$, we have that $d^{+}(x, A) \leqslant \beta n$, so

$$
d^{+}(x)=d^{+}(x, A)+d^{+}(x, \bar{A})<(1 / 2-c) n,
$$

a contradiction to the minimum semidegree condition.

Proof of Lemma 14. Define $\xi, \beta$ and $\omega$ so that

$$
1 / n \ll c \ll \mu, \alpha \ll \xi \ll \beta \ll \omega \ll \gamma, \eta<1 \text {. }
$$

By assumption, there exists $\mathcal{P}$ a non-trivial $\eta$-partition of $V(G)$ such that $L_{\mathcal{P}}^{\mu}(H(G))$ is 2 -transferral-free and $A \in \mathcal{P}$ such that $\operatorname{cyc}(A, A, \bar{A}) \leqslant \alpha n^{3}$. Since $\mathcal{P}$ is an $\eta$-partition, we have that

$$
|A| \geqslant \eta n \text {. }
$$

For (possibly overlapping) subsets $U_{1}, U_{2}, U_{3}, U_{4}$ of $V$, let $L\left(U_{1}, U_{2}, U_{3}, U_{4}\right)$ be the collection of 4 -sets $\left\{u_{1}, u_{2}, u_{3}, u_{4}\right\}$ such that $u_{i} \in U_{i}$ for $i \in[4]$ and both $u_{1} u_{2} u_{3}$ and $u_{2} u_{3} u_{4}$ are cyclic triangles. We can assume that

$$
|L(A, V, V, \bar{A})| \leqslant \xi n^{4}
$$

because otherwise, since $|\mathcal{P}| \leqslant 1 / \eta$, there would exist $B, C \in \mathcal{P}$ and $D \in \mathcal{P}-A$ such that $|L(A, B, C, D)| \geqslant \eta^{3} \cdot \xi n^{4} \geqslant 3 \mu n^{4}$, which would in turn imply that both $c(A, B, C) \geqslant \mu n^{3}$ and $c(B, C, D) \geqslant \mu n^{3}$, which contradicts the fact that $L_{\mathcal{P}}^{\mu}(H(G))$ is 2-transferral-free.

Define $S^{\sigma}=\operatorname{SN}_{\beta}^{\sigma}(A)$ for $\sigma \in\{+,-\}$, and let $S=V \backslash\left(S^{+} \cup S^{-} \cup A\right)$. Lemma 23 implies that

$$
|S| \leqslant 2 \xi n
$$


and that

$$
|A| \leqslant(1 / 3+\xi) n \text {. }
$$

For $\sigma \in\{+,-\}$, suppose that there exists $x y z$ a triangle with $x y \in E\left(S^{\sigma}\right)$ and $z \in S^{-\sigma}$, i.e., $x y z$ is one of the triangles that is counted in $\operatorname{cyc}\left(S^{\sigma}, S^{\sigma}, S^{-\sigma}\right)$. If $\sigma=+$, then for every $w \in N^{-,+}(y z, A)$, we have $\{w, y, z, x\} \in L\left(A, S^{+}, S^{-}, S^{+}\right)$, and if $\sigma=-$, then for every $w \in N^{-,+}(z x, A)$, we have $\{w, x, z, y\} \in L\left(A, S^{-}, S^{+}, S^{-}\right)$. Therefore, because $d^{-\sigma}(x, A), d^{-\sigma}(y, A), d^{\sigma}(z, A) \geqslant|A|-\beta n,(20)$ implies that

$$
(|A|-2 \beta n) \cdot c\left(S^{\sigma}, S^{\sigma}, S^{-\sigma}\right) \leqslant\left|L\left(A, S^{\sigma}, S^{-\sigma}, S^{\sigma}\right)\right| \leqslant \xi n^{4} .
$$

Therefore, with (19), we have

$$
\operatorname{cyc}\left(S^{\sigma}, S^{\sigma}, S^{-\sigma}\right) \leqslant \beta n^{3} .
$$

Furthermore,

$$
\operatorname{cyc}\left(S^{\sigma}, S^{\sigma}, A\right) \leqslant 2 \beta n \cdot\left(\begin{array}{c}
\left|S^{\sigma}\right| \\
2
\end{array}\right) \leqslant \beta n^{3},
$$

and, because (21) implies that

$$
\operatorname{cyc}\left(S^{\sigma}, S^{\sigma}, S\right) \leqslant 2 \beta n^{3},
$$

we can deduce that

$$
\operatorname{cyc}\left(S^{\sigma}, S^{\sigma}, \overline{S^{\sigma}}\right)=\operatorname{cyc}\left(S^{\sigma}, S^{\sigma}, S^{-\sigma}\right)+\operatorname{cyc}\left(S^{\sigma}, S^{\sigma}, A\right)+\operatorname{cyc}\left(S^{\sigma}, S^{\sigma}, S\right) \leqslant 4 \beta n^{3} .
$$

Applying Lemma 23 with $S^{\sigma}, 4 \beta$ and $\omega$ playing the roles of $A, \alpha$ and $\xi$, respectively, implies that

$$
\left|S^{+}\right|,\left|S^{-}\right| \leqslant(1 / 3+\omega) n \text {. }
$$

Let $A^{\prime}=A \cup S$ and note that $\left\{A^{\prime}, S^{+}, S^{-}\right\}$is a partition of $V(G)$, and, since (21), (22) and (23) imply that $\left|A^{\prime}\right|,\left|S^{+}\right|,\left|S^{-}\right| \leqslant n / 3+\omega n$, we have

$$
\left|A^{\prime}\right|,\left|S^{+}\right|,\left|S^{-}\right|=n / 3 \pm 2 \omega n .
$$

By (21), we have that

$$
d^{-\sigma}\left(v, A^{\prime}\right) \geqslant|A|-\beta n \geqslant\left|A^{\prime}\right|-\omega n \text { for every } v \in S^{\sigma},
$$

which implies that

$$
e^{\sigma}\left(S^{\sigma}, A^{\prime}\right) \leqslant\left|S^{\sigma}\right| \cdot \omega n \leqslant \omega n^{2},
$$

and, also, with Lemma 16, that

$$
e^{+}\left(S^{+}, \overline{S^{+}}\right) \geqslant e^{+}\left(\overline{S^{+}}, S^{+}\right)-c n^{2} \geqslant e^{+}\left(A^{\prime}, S^{+}\right)-c n^{2} \geqslant\left(\left|A^{\prime}\right|-\omega n\right)\left|S^{+}\right|-c n^{2} .
$$

Combining this with (24) and (25) gives us that

$$
e^{+}\left(S^{+}, S^{-}\right)=e^{+}\left(S^{+}, \overline{S^{+}}\right)-e^{+}\left(S^{+}, A^{\prime}\right) \geqslant\left(\left|A^{\prime}\right|-\omega n\right)\left|S^{+}\right|-2 \omega n^{2} \geqslant\left|S^{-}\right|\left|S^{+}\right|-\gamma n^{2},
$$

so $e^{+}\left(S^{-}, S^{+}\right) \leqslant \gamma n^{2}$, which, with $(24)$ and (25), implies that $G$ is $\gamma$-extremal. 


\subsection{Proof of Theorem 7 - The Extremal Case}

We use the following tripartite Corrádi-Hajnal theorem from [9], although we do not require its full strength. In fact, we only use it for the corollary which follows that could easily be proved directly. The multipartite Hajnal-Szemerédi theorem has been heavily studied by many researchers, for example, see [4, 12, 14, 18, 19].

Theorem 24 (Johansson $2000[9])$. Let $G=(V, E)$ be a graph. If there exists $\left\{V_{1}, V_{2}, V_{3}\right\}$ a partition of $V$ such that $\left|V_{1}\right|=\left|V_{2}\right|=\left|V_{3}\right|=m$ and, for every $i \in[3]$ and $v \in V_{i}$, $\left|N(v) \cap V_{i+1}\right|,\left|N(v) \cap V_{i-1}\right| \geqslant \frac{2}{3} m+\sqrt{m}$ then $G$ has a triangle factor.

Corollary 25. Suppose that $0<1 / n \ll c, \xi \ll 1, n$ is a multiple of 3 , and $G=(V, E)$ is an oriented graph on $n$ vertices such that $\delta^{0}(G) \geqslant\left(\frac{1}{2}-c\right) n$. If there exists a partition $\left\{V_{1}, V_{2}, V_{3}\right\}$ of $V$ such that $\left|V_{1}\right| \equiv\left|V_{2}\right| \equiv\left|V_{3}\right|(\bmod 3)$ and, for every $i \in[3]$ and $v \in V_{i}$, we have $d^{+}\left(v, V_{i+1}\right), d^{-}\left(v, V_{i-1}\right) \geqslant\left(\frac{1}{3}-\xi\right) n$, then $G$ has a cyclic triangle factor.

Proof. Assume without loss of generality that $\left|V_{1}\right| \leqslant\left|V_{2}\right| \leqslant\left|V_{3}\right|$. For every $i \in[3]$, the degree condition implies that $\left|V_{i}\right|=(1 / 3 \pm 2 \xi) n$, so

$$
\delta^{0}\left(G\left[V_{i}\right]\right) \geqslant(1 / 2-c) n-3 \xi n-(1 / 3+2 \xi) n \geqslant(1 / 2-3 c-18 \xi)\left|V_{i}\right| .
$$

Therefore, by Lemma 17, we can greedily find a collection $\mathcal{C}_{2}$ of $\left(\left|V_{2}\right|-\left|V_{1}\right|\right) / 3 \leqslant 2 \xi n$ vertex-disjoint cyclic-triangles in $G\left[V_{2}\right]$, and a collection $\mathcal{C}_{3}$ of $\left(\left|V_{3}\right|-\left|V_{1}\right|\right) / 3 \leqslant 2 \xi n$ vertexdisjoint cyclic-triangles in $G\left[V_{3}\right]$. Let $H$ be the oriented graph induced by $V \backslash V\left(\mathcal{C}_{2} \cup \mathcal{C}_{3}\right)$. Theorem 24 implies that there exists a triangle factor of the spanning subgraph of the simple graph underlying $H$ that contains the edges that correspond to the edges of $H$ that are directed either from $V_{1}$ to $V_{2}$, from $V_{2}$ to $V_{3}$, or from $V_{3}$ to $V_{1}$. This triangle factor corresponds to a cyclic triangle factor $\mathcal{C}$ of $H$. Then $\mathcal{C} \cup \mathcal{C}_{2} \cup \mathcal{C}_{3}$ is a triangle factor of $G$.

Proof of Lemma \%. If $G$ has a divisibility barrier, then, by the argument presented before Definition 3, $G$ does not contain a cyclic triangle factor. For the other direction, assume that $G$ does not have a divisibility barrier. Since $G$ is $\gamma$-extremal, there exists a partition $\left\{V_{1}, V_{2}, V_{3}\right\}$ of $V$ such that for every $i \in[3],\left|V_{i}\right|=(1 / 3 \pm \gamma) n$ and $e^{-}\left(V_{i}, V_{i+1}\right) \leqslant \gamma n^{2}$. Introduce new constants $\alpha$ and $\beta$, so that

$$
0<c, \gamma \ll \alpha \ll \beta \ll 1
$$

Let

$$
U_{i}=\mathrm{SN}_{\beta}^{-}\left(V_{i+1}\right) \cap \mathrm{SN}_{\beta}^{+}\left(V_{i-1}\right) \quad \text { for } i \in[3],
$$

and let $U_{0}=V \backslash\left(U_{1} \cup U_{2} \cup U_{3}\right)$. Note that $\left\{U_{0}, U_{1}, U_{2}, U_{3}\right\}$ is a partition of $V$. For every $i \in[3]$ and every $v \in V_{i} \backslash U_{i}$, at least one of $d^{-}\left(v, V_{i+1}\right)$ or $d^{+}\left(v, V_{i-1}\right)$ is at least $\beta n-2 c n \geqslant \beta n / 2$, so

$$
\frac{1}{2} \sum_{i=1}^{3}\left|V_{i} \backslash U_{i}\right| \cdot \beta n \leqslant 2 e^{-}\left(V_{1}, V_{2}\right)+2 e^{-}\left(V_{2}, V_{3}\right)+2 e^{-}\left(V_{3}, V_{1}\right) \leqslant 6 \gamma n^{2},
$$


and we have that

$$
\left|U_{0}\right| \leqslant \sum_{i=1}^{3}\left|V_{i} \backslash U_{i}\right| \leqslant \alpha n .
$$

Suppose that $\left|U_{0}\right| \geqslant 1$. By (26) and Lemma 17, we can greedily find a (possibly empty) collection $\mathcal{C}$ of vertex-disjoint cyclic-triangles such that

$$
|V(\mathcal{C})| \leqslant 3\left(\left|U_{0}\right|-1\right) \leqslant 3 \alpha n
$$

and $\left|U_{0} \cap V(\mathcal{C})\right|=\left|U_{0}\right|-1$. Let $u$ be the vertex in $U_{0}$ that is not covered by $\mathcal{C}$, i.e., $\{u\}=U_{0} \backslash V(\mathcal{C})$. Let $G^{\prime}=G-V(\mathcal{C})$, and, for $i \in[3]$, let $W_{i}=U_{i} \backslash V(\mathcal{C})$. Note that, by (26) and (27),

$$
\left|V_{i} \backslash W_{i}\right| \leqslant 4 \alpha n \quad \text { for } i \in[3] .
$$

Because $\left|G^{\prime}\right|=|V \backslash V(\mathcal{C})|$ is divisible by 3 , we have that $\left|W_{1}\right|+\left|W_{2}\right|+\left|W_{3}\right| \equiv 2(\bmod 3)$, and we can assume without loss of generality that $\left|W_{2}\right| \equiv\left|W_{3}\right|(\bmod 3)$. Fix $x \in\{0,1,2\}$ so that

$$
\left|W_{1}\right| \equiv x+2 \text { and }\left|W_{2}\right| \equiv\left|W_{3}\right| \equiv x \quad(\bmod 3) .
$$

If there exists a triangle $T$ in $G^{\prime}$ such that $u \in V(T)$ and either $\left|V(T) \cap W_{1}\right|=2$ or $\left|V(T) \cap W_{2}\right|=\left|V(T) \cap W_{3}\right|=1$, then we can complete a cyclic triangle factor of $G$ using Corollary 25, so assume the contrary.

Let $W_{1}^{+}=N^{+}\left(u, W_{1}\right), W_{1}^{-}=N^{-}\left(u, W_{1}\right)$ and $W_{1}^{0}=W_{1} \backslash N(u)$. We have that $e^{+}\left(W_{1}^{+}, W_{1}^{-}\right)=0$ by assumption, and, by the minimum semidegree condition,

$$
\left|W_{1}^{0}\right| \leqslant 2 c n .
$$

Assume that $\left|W_{1}^{-}\right| \leqslant\left|W_{1}^{+}\right|$. If $W_{1}^{-} \neq \varnothing$, then there exists $x \in W_{1}^{-}$such that

$$
d^{-}\left(x, W_{1}^{-}\right)<\left|W_{1}^{-}\right| / 2 \leqslant\left|W_{1}\right| / 4,
$$

so, by (28) and (29),

$$
d^{-}\left(x, W_{1}^{+}\right) \geqslant \delta^{0}(G)-\left(d^{-}\left(x, W_{1}^{-} \cap V_{1}\right)+\left|W_{1}^{0} \cap V_{1}\right|+\left|V_{1} \backslash W_{1}\right|+d^{-}\left(x, V_{2}\right)+\left|V_{3}\right|\right)>0,
$$

a contradiction. Therefore, we have $W_{1}^{-}=\varnothing$. This, with (28) and (29), implies that

$$
d^{+}\left(u, V_{1}\right) \geqslant\left|W_{1} \cap V_{1}\right|-\left|W_{1}^{0}\right|=\left|V_{1}\right|-\left|V_{1} \backslash W_{1}\right|-\left|W_{1}^{0}\right| \geqslant\left|V_{1}\right|-\beta n,
$$

and

$$
d^{-}\left(u, W_{3} \cap V_{3}\right) \geqslant \delta^{0}(G)-\left(d^{-}\left(u, V_{1}\right)+\left|V_{2}\right|+\left|V_{3} \backslash W_{3}\right|\right)>(1 / 6-2 \beta) n .
$$

By the definition of $W_{2}$, this implies that every vertex in $W_{2}$ has an out-neighbor in $N^{-}\left(u, W_{3} \cap V_{3}\right)$. Therefore, because $e^{+}\left(N^{+}\left(u, W_{2}\right), N^{-}\left(u, W_{3}\right)\right)=0$ by assumption, we have that $N^{+}\left(u, W_{2}\right)=\varnothing$, and, by a computation similar to (30)

$$
d^{-}\left(u, V_{2}\right) \geqslant\left|W_{2} \cap V_{2}\right|-\left|W_{2} \backslash N(u)\right|=\left|V_{2}\right|-\left|V_{2} \backslash W_{2}\right|-\left|W_{2} \backslash N(u)\right| \geqslant\left|V_{2}\right|-\beta n,
$$


and this with (30) implies that $u \in U_{3}$, a contradiction to the fact that $u \in U_{0}$. By a similar argument, if $\left|W_{1}^{-}\right| \geqslant\left|W_{1}^{+}\right|$, one can show that $W_{1}^{+}=\varnothing$ and then $u \in U_{2}$, a contradiction.

Now suppose that $\left|U_{0}\right|=0$. By Corollary 25, we are done if $\left|U_{1}\right| \equiv\left|U_{2}\right| \equiv\left|U_{3}\right|$ (mod 3), so assume the contrary. If $G$ has no divisibility barriers, then $|V|$ is divisible by 3 , and, without loss of generality we can assume that there exists an edge $a b$ such that $a \in U_{2}$ and $b \in U_{1}$. Because $|V|$ is divisible by 3 , we can fix $x \in\{0,1,2\}$ and $y \in\{1,2\}$ so that

$$
\left|U_{1}\right| \equiv x,\left|U_{2}\right| \equiv x+y, \text { and }\left|U_{3}\right|=x-y \quad(\bmod 3)
$$

Note that, by (26),

$$
d^{+}\left(b, U_{1}\right) \geqslant \delta^{0}(G)-\left(\left|V_{1} \backslash U_{1}\right|+\left|V_{2}\right|+d^{+}\left(b, V_{3}\right)\right) \geqslant(1 / 6-2 \beta) n,
$$

and a similar bound holds for $d^{-}\left(a, U_{2}\right)$, so there exists a vertex $c \in U_{2} \cap N^{+}(b) \cap N^{-}(a)$, and a vertex $d \in U_{1} \cap N^{+}(b) \cap N^{-}(a)$. Hence $T_{1}=a b c$ and $T_{2}=a b d$ are cyclic triangles and, for $j \in\{1,2\}$,

$$
\left|V\left(T_{j}\right) \cap U_{1}\right| \equiv j \text { and }\left|V\left(T_{j}\right) \cap U_{2}\right| \equiv 2 j \quad(\bmod 3) .
$$

Therefore, Corollary 25 implies that there exists a cyclic triangle factor $\mathcal{C}$ of $G-T_{y}$, and $\mathcal{C} \cup\left\{T_{y}\right\}$ is a cyclic triangle factor of $G$.

\section{References}

[1] J. Balogh, A. Lo, and T. Molla, Transitive triangle tilings in oriented graphs, J. Combin. Theory Ser. B, 124 (2017), 64-87.

[2] L. Caccetta and R. Häggkvist, On minimal digraphs with given girth, in: Proceeding of the Ninth Southeastern Conference on Combinatorics, Graph Theory and Computing, Florida Atlantic Univ., Boca Raton, Fla., 1978, in: Congr. Numer., vol. XXI, Utilitas Math., Winnipeg, Man., 1978, 181-187.

[3] K. Corrádi and A. Hajnal, On the maximal number of independent circuits in a graph, Acta Mathematica Hungarica 14 (1963), no. 3, 423-439.

[4] B. Csaba and M. Mydlarz, Approximate multipartite version of the Hajnal-Szemerédi theorem, J. Combin. Theory, Ser. B 102 (2012), no. 2, 395-410.

[5] B. Cuckler, On the number of short cycles in regular tournaments, unpublished manuscript (2008).

[6] A. Czygrinow, DeBiasio L., Molla T., and A. Treglown, Tiling directed graphs with tournaments, Forum Math. Sigma. 6 (2018), e2.

[7] A. Hajnal and E. Szemerédi, Proof of a conjecture of P. Erdős, Combinatorial Theory and Its Application 2 (1970), 601-623.

[8] J. Han, Decision problem for perfect matchings in dense k-uniform hypergraphs, Trans. Amer. Math. Soc. 369 (2017), no. 7, 5197-5218. 
[9] R. Johansson, Triangle-factors in a balanced blown-up triangle, Discrete Math. 211 (2000), no. 1-3, 249-254.

[10] P. Keevash, F. Knox, and R. Mycroft, Polynomial-time perfect matchings in dense hypergraphs, Adv. Math. 269 (2015), 265-334.

[11] P. Keevash and R. Mycroft, A geometric theory for hypergraph matching, Mem. Amer. Math. Soc. 233 (2015), no. 1098.

[12] _ A multipartite Hajnal-Szemerédi theorem, J. Combin. Theory Ser. B 114 (2015), 187-236.

[13] P. Keevash and B. Sudakov, Triangle packings and 1-factors in oriented graphs, J. Combin. Theory Ser. B 99 (2009), no. 4, 709-727.

[14] A. Lo and K. Markström, A multipartite version of the Hajnal-Szemerédi theorem for graphs and hypergraphs, Combin. Probab. Comput. 22 (2013), no. 1, 97-111.

[15] , Minimum codegree threshold for $\left(K_{3}^{4}-e\right)$-factors, J. Combin. Theory Ser. A 120 (2013), no. 3, 708-721.

[16] _ Perfect matchings in 3-partite 3-uniform hypergraphs, J. Combin. Theory Ser. A 127 (2014), 22-57.

[17] _ F-factors in hypergraphs via absorption, Graphs Combin. 31 (2015), no. 3, 679-712.

[18] C. Magyar and R. Martin, Tripartite version of the Corrádi-Hajnal theorem, Discrete Math. 254 (2002), no. 1-3, 289-308.

[19] R. Martin and E. Szemerédi, Quadripartite version of the Hajnal-Szemerédi theorem, Discrete Math. 308 (2008), no. 19, 4337-4360.

[20] V. Rödl, A. Ruciński, and E. Szemerédi, A Dirac-type theorem for 3-uniform hypergraphs, Combin. Probab. Comput. 15 (2006), no. 1-2, 229-251.

[21] A. Treglown, A note on some embedding problems for oriented graphs, J. Graph Theory 69 (2012), no. 3, 330-336.

[22] H. Wang, Independent directed triangles in a directed graph, Graphs Combin. 16 (2000), no. 4, 453-462.

[23] R. Yuster, Tiling transitive tournaments and their blow-ups, Order 20 (2003), no. 2, 121-133.

[24] R. Yuster, Combinatorial and computational aspects of graph packing and graph decomposition, Comput. Sci. Rev. 1 (2007), no. 1, 12-26. 